\title{
On the extraction of the boundary conditions and the boundary ports in second-order field theories
}

\author{
Markus Schöberl and Kurt Schlacher
}

January 26, 2018

\begin{abstract}
Institute of Automatic Control and Control Systems Technology, Johannes Kepler University, Altenbergerstr. 69, 4040 Linz, Austria
\end{abstract}

\begin{abstract}
In this paper we consider second-order field theories in a variational setting. From the variational principle the Euler-Lagrange equations follow in an unambiguous way, but it is well known that this is not true for the Cartan form. This has also consequences on the derivation of the boundary conditions when non trivial variations are allowed on the boundary. By posing extra conditions on the set of possible boundary terms we exploit the degree of freedom in the Cartan form to extract physical meaningful boundary expressions. The same mathematical machinery will be applied to derive the boundary ports in a Hamiltonian representation of the partial differential equations which is crucial for energy based control approaches. Our results will be visualized for mechanical systems such as beam and plate models.
\end{abstract}

\section{Introduction}

Many distributed-parameter systems described by partial differential equations (pdes) allow for a variational formulation such that they can be analyzed based on the corresponding Lagrangian density. In a geometric setting the Euler-Lagrange equations are constructed by using the so-called Cartan form, which provides a coordinate free way to perform integration by parts, see e.g. [1, 7, 10, 18, 19] and references therein. It is well-known that a unique formulation for the Euler-Lagrange equations can be found but this is not true for the Cartan form when the variational problem involves derivative variables of second-order or higher and more than one independent coordinate. This is connected to the commutativity of repeated partial differentiation. In the literature many attempts have been made to fix the Cartan form also in the higher-order field theories, e.g. by using connections, see e.g [5] or to avoid the ambiguities by using different approaches e.g. the Skinner and Rusk formalism, see e.g. 2]. Another consequence of the ambiguities of the Cartan form (i.e. the need for repeated integration by parts) is the fact that also the terms in the boundary integral are affected by this non-uniqueness, when non-trivial variations on the boundary are explicitly allowed. In the mathematical physicist literature this effect is usually ignored and only trivial boundary conditions are considered, but e.g. for mechanical structures such as beams, plates or shells it is of great importance to allow for non trivial boundary terms and consequently also for non-zero energy flow over the boundary, especially for engineering applications, e.g. for control purposes and especially for boundary control systems.

In this paper we consider non-trivial variations on the boundary and we restrict ourselves to second-order field theories. The case of second-order field theories is in some sense special, see e.g. 9, 17, 18, but to our best knowledge a systematic derivation of non-trivial boundary conditions based on the Cartan form has not been reported in the literature so far. In 9] it is shown by evaluating the variational principle (using integration by parts) that the boundary term is indeed connected with the Cartan form but the extraction of the meaningful boundary conditions is not 
discussed in this setting. In [15] natural boundary conditions for higher-order field theories in a variational setting are discussed based on flag fibrations and again using integration by parts.

We demonstrate that by posing extra conditions on the set of possible boundary terms, one is able to derive the physically admissible boundary conditions which will be demonstrated for mechanical systems where the Lagrangian depends on second-order derivatives. To this end, we use the ambiguities in constructing the Cartan form and we exploit the special structure of the boundary manifold of the underlying variational problem, where we make use of an adapted coordinate system as it has been suggested e.g. in [3, 15] to present our result in a compact and simple manner. Some preliminary work has been reported in [22] and [23].

Furthermore, we will focus on partial differential equations (pdes) that allow for a Hamiltonian formulation. Given pdes in a Lagrangian setting, different approaches to construct Hamiltonian counterparts have been discussed in the literature including symplectic, polysymplectic and multisymplectic cases see, e.g. [6, 8, 16]. From a control and a system theoretic point of view the class of port-Hamiltonian systems with dissipation [26] is a very prominent system class, which has been extended from the lumped-parameter case (systems described by ordinary differential equations) to the distributed-parameter pde case. However, different port-Hamiltonian formulations exist, see e.g. [27, 13, 11, 12] for an approach based on a Stokes-Dirac structure whereas in [4, 20, 23, 24, 25] an alternative strategy based on jet-bundles has been considered. In this paper we will consider the jet-bundle approach and apply the theory of Cartan forms to be able to extract the correct boundary ports when the Hamiltonian depends on second-order derivative variables in a formal and systematic way.

The main contribution of the paper is i) to provide a simple and algorithmic procedure to derive the physical admissible boundary conditions for variational problems by means of the Cartan form where the Lagrangian depends on second-order derivative variables and ii) to apply this mathematical machinery developed for the Lagrangian scenario to a port-Hamiltonian representation in order to analyze the power balance relation. The paper is organized as follows. In Section 2 some geometric fundamentals are presented and in the third section we discuss first-order theories where we consider the well-known case of the Cartan form approach to derive the pdes and the boundary terms in a Lagrangian setting. Then, Section 4 is devoted to the second-order case in the Lagrangian framework, where we show how to extract the boundary conditions by using the presented machinery. In Section 5 we discuss the Euler-Bernoulli beam and the Kirchhoff plate in the Lagrangian framework. In the sixth section the port-Hamiltonian perspective is analyzed within this mathematical setting and the Kirchhoff plate example is rearranged in a Hamiltonian formulation to make the power ports visible.

\section{Notation and Preliminaries}

In this paper we will apply differential geometric methods, in particular we use jet-bundles and induced geometric objects, see [19] for an extensive treatise. We use tensor notation and especially Einsteins convention on sums where we will not indicate the range of the indices when they are clear from the context. Given a manifold $\mathcal{M}$ with local coordinates $\left(x^{\alpha}\right)$ where $\alpha=1, \ldots, \operatorname{dim}(\mathcal{M})$ we denote with $\mathcal{T}(\mathcal{M}) \rightarrow \mathcal{M}$ and $\mathcal{T}^{*}(\mathcal{M}) \rightarrow \mathcal{M}$ the tangent bundle and the cotangent bundle, respectively. Local coordinates for $\mathcal{T}(\mathcal{M})$ and $\mathcal{T}^{*}(\mathcal{M})$ are $\left(x^{\alpha}, \dot{x}^{\alpha}\right)$ as well as $\left(x^{\alpha}, \dot{x}_{\alpha}\right)$ with respect to the holonomic fibre basis $\left\{\partial_{\alpha}\right\}$ and $\left\{\mathrm{d} x^{\alpha}\right\}$. Furthermore, we use $\wedge$ for the exterior product (wedge product), $\mathrm{d}$ is the exterior derivative and $\rfloor$ denotes the natural contraction between tensor fields. With $\mathrm{L}_{v}(\omega)$ we denote the Lie-derivative of the geometric object $\omega$ (e.g. a differential form) with respect to the vector field $v$. Furthermore, $C^{\infty}(\cdot)$ denotes the set of smooth functions on the corresponding manifold.

Let $\mathcal{X} \stackrel{\pi}{\rightarrow} \mathcal{D}$ be a bundle with $\operatorname{dim}(\mathcal{X})=m+r$ where $\mathcal{D}$ is an orientable $r$-dimensional manifold. Local coordinates for $\mathcal{X}$ adapted to the fibration are $\left(X^{i}, x^{\alpha}\right)$ with $x^{\alpha}, \alpha=1, \ldots, m$ where $X^{i}, i=$ $1, \ldots, r$ is a coordinate chart for $\mathcal{D}$. We fix the volume form $\Omega$ on the manifold $\mathcal{D}$ as $\Omega=\mathrm{d} X^{1} \wedge \ldots \wedge$ $\mathrm{d} X^{r}$ and we set $\left.\Omega_{i}=\partial_{i}\right\rfloor \Omega$. Let $\sigma$ be a section of the bundle $\mathcal{X} \rightarrow \mathcal{D}$, i.e. a map $\sigma: \mathcal{D} \rightarrow \mathcal{X}$ with coordinate representation $\sigma\left(X^{i}\right)=\left(X^{i}, \sigma^{\alpha}\left(X^{i}\right)\right)$. The set of sections of the bundle $\mathcal{X} \stackrel{\pi}{\rightarrow} \mathcal{D}$ will be 
denoted as $\Gamma(\pi)$. Then the $k$-th order partial derivatives of $\sigma^{\alpha}$ are

$$
\frac{\partial^{k} \sigma^{\alpha}}{\left(\partial X^{1}\right)^{j_{1}} \cdots\left(\partial X^{r}\right)^{j_{r}}}=\partial_{J} \sigma^{\alpha}
$$

where $J$ is an ordered multi-index with $\sum j_{i}=k=\# J$. The components of the multi-index $J$ are $J(i)$ and $1_{k}$ is the special multi index defined as $J(i)=\delta_{k}^{i}$ such that all components of $1_{k}$ are zero except the $k$-th entry which is one. Two multi-indices $J$ and $I$ can be added and subtracted component wise, where the result of a subtraction may not be a multi-index.

Given two sections, such that at a point $p \in \mathcal{D}$ the sections and their partial derivatives up to order $k$ coincide, these sections are called $k$-equivalent. Given now a smooth local section $\sigma \in \Gamma_{p}(\pi)$ at point $p \in \mathcal{D}$, the equivalence class of $n$-equivalent sections that contains $\sigma$ is denoted $j_{p}^{n}(\sigma)$ (called the $n$-jet at $p$ ) and the set of all these $n$-jets is the $n$-th order jet-manifold $\mathcal{J}^{n}(\mathcal{X})$ with adapted coordinates $\left(X^{i}, x_{J}^{\alpha}\right)$ with $0 \leq \# J \leq n$. Furthermore, we have $\pi_{k, l}: \mathcal{J}^{k}(\mathcal{X}) \rightarrow \mathcal{J}^{l}(\mathcal{X})$ for $k>l$ as well as $\pi_{k, 0}: \mathcal{J}^{k}(\mathcal{X}) \rightarrow \mathcal{X}$ and $\pi_{k}: \mathcal{J}^{k}(\mathcal{X}) \rightarrow \mathcal{D}$. The tangent and the cotangent bundles of $\mathcal{J}^{k}(\mathcal{X})$ can be easily constructed and the holonomic fibre bases for $\mathcal{T}\left(\mathcal{J}^{k}(\mathcal{X})\right)$ and $\mathcal{T}^{*}\left(\mathcal{J}^{k}(\mathcal{X})\right.$ ) read as $\left\{\partial_{i}, \partial_{\alpha}^{J}\right\}$ and $\left\{\mathrm{d} X^{i}, \mathrm{~d} x_{J}^{\alpha}\right\}$, where $0 \leq \# J \leq k$, respectively, with $\partial_{i}=\partial / \partial X^{i}$ and $\partial_{\alpha}^{J}=\partial / \partial x_{J}^{\alpha}$.

The total derivative $d_{1_{i}}$ with respect to the independent variable $X^{i}$ reads as

$$
d_{1_{i}}=\partial_{i}+x_{J+1_{i}}^{\alpha} \partial_{\alpha}^{J}
$$

and $\left(d_{1_{i}} f\right) \circ j^{n+1}(\sigma)=\partial_{i}\left(f \circ j^{n}(\sigma)\right)$ is met by construction for all $f \in C^{\infty}\left(\mathcal{J}^{n}(\mathcal{X})\right)$ and $\sigma \in \Gamma(\pi)$. The $k$-th order total derivative meets $d_{1_{i}} \in \pi_{k+1, k}^{*}\left(\mathcal{T}\left(\mathcal{J}^{k}(\mathcal{X})\right)\right)$. The dual elements to the total derivatives are the so-called contact forms. In local coordinates we have

$$
\omega_{J}^{\alpha}=\mathrm{d} x_{J}^{\alpha}-x_{J+1_{i}}^{\alpha} \mathrm{d} X^{i}, \quad \# J \geq 0,
$$

where a $k$-th order contact form meets $\omega_{J}^{\alpha} \in \pi_{k+1, k}^{*}\left(\mathcal{T}^{*}\left(\mathcal{J}^{k}(\mathcal{X})\right)\right)$ with $\# J=k$ and $j^{k}(\sigma)^{*}\left(\omega_{J}^{\alpha}\right)=0$ is met for $\sigma \in \Gamma(\pi)$. Furthermore, based on the structure of the underlying fibre bundle the operator of horizontalisation hor, see e.g. [10, can be introduced as

$$
\operatorname{hor}\left(\mathrm{d} X^{i}\right)=\mathrm{d} X^{i}, \operatorname{hor}\left(\mathrm{d} x_{J}^{\alpha}\right)=x_{J+1_{i}}^{\alpha} \mathrm{d} X^{i}
$$

which has the property that hor $\left(\omega_{J}^{\alpha}\right)=0$.

The geometric version of the calculus of variations rests on the use of so-called variational vector fields $\eta$, whose flow is used to perform variations of sections $\sigma \in \Gamma(\pi)$, where we consider the special case of vertical variations. Hence, these vector fields $\eta$ are tangent to the fibration of $\mathcal{X} \stackrel{\pi}{\rightarrow} \mathcal{D}$ and they are referred to as vertical vector fields, i.e. $\eta \in \mathcal{V}(\mathcal{X})$ with the coordinate representation $\eta=\eta^{\alpha}(X, x) \partial_{\alpha}$, i.e. meeting $\dot{X}=0$. Furthermore, we need the $n$-th jet-prolongation of a vertical vector field $\eta \in \mathcal{V}(\mathcal{X})$ which is given as

$$
j^{n}(\eta)=\eta^{\alpha} \partial_{\alpha}+d_{J}\left(\eta^{\alpha}\right) \partial_{\alpha}^{J}, \quad d_{J}=\left(d_{1_{1}}\right)^{j_{1}} \ldots\left(d_{1_{r}}\right)^{j_{r}}
$$

with $1 \leq \# J \leq n$, see e.g. [16, 19. It is well-known that these jet-prolongations of vertical vector fields will play a prominent role when formulating a variation problem as recapitulated next, see again [19].

Let us consider a variational problem on the bundle $\mathcal{X} \stackrel{\pi}{\rightarrow} \mathcal{D}$ where the Lagrangian is of the form $\mathfrak{L}=\mathcal{L} \Omega$ with $\mathcal{L} \in C^{\infty}\left(\mathcal{J}^{k}(\mathcal{X})\right)$ and induces a functional $L(\sigma)=\int_{\mathcal{S}} j^{k}(\sigma)^{*} \mathfrak{L}$ for sections $\sigma \in \Gamma(\pi)$ where $\mathcal{S}$ is a submanifold of $\mathcal{D}$. In the calculus of variations, the task is to find so-called critical sections $\sigma$ that extremize the functional $L(\sigma)$. To find these critical sections we will consider vertical variations $\phi_{\epsilon}$ (fiber preserving maps described by a flow). A critical section $\sigma$ meets

$$
d_{\epsilon}\left(\left.\int_{\mathcal{S}}\left(j^{k}\left(\phi_{\epsilon} \circ \sigma\right)^{*} \mathfrak{L}\right)\right|_{\epsilon=0}=0,\right.
$$

which is equivalent to

$$
\int_{\mathcal{S}} j^{k}(\sigma)^{*}\left(\mathrm{~L}_{j^{k}(\eta)}(\mathfrak{L})\right)=0
$$

see e.g. [19], where $\eta$ is the infinitesimal generator of the flow $\phi_{\epsilon}$. 


\section{First-order Case}

In this section we will recapitulate the well-known facts of a variational problem for first-order Lagrangians, i.e. $\mathcal{L} \in C^{\infty}\left(\mathcal{J}^{1}(\mathcal{X})\right)$. However, contrary to most of the differential geometric approaches in the literature we allow for non-trivial boundary conditions and we will demonstrate how the Cartan form is connected to these boundary terms.

Remark 1. These non-trivial boundary terms play a crucial role in many applications, e.g. in engineering, where systems can be interconnected via their boundaries, see e.g. [4, 20, 21, 24, 25] for this port based approach, or where actuators directly influence the boundary variables - boundary control systems.

This introductory section serves mainly as a reminder but it should clarify some of the ideas that are then used in second-order case $k=2$ in the forthcoming section. Let us consider the equation (44) for $k=1$ then using

$$
\left.\left.\mathrm{L}_{j^{1}(\eta)}(\mathfrak{L})=j^{1}(\eta)\right\rfloor \mathrm{d} \mathfrak{L}+\mathrm{d}\left(j^{1}(\eta)\right\rfloor \mathfrak{L}\right)
$$

there is a natural decomposition into a domain and a boundary term, as by Stokes' theorem $\left.\mathrm{d}\left(j^{1}(\eta)\right\rfloor \mathfrak{L}\right)$ contributes to the boundary. Unfortunately, we cannot pick the domain conditions directly as $\left.j^{1}(\eta)\right\rfloor \mathrm{d} \mathfrak{L}$ may depend on derivatives of the variational field $\eta$ that induces further terms on the boundary. However, if we are able to reformulate the problem such that no prolongation of $\eta$ is necessary we are done. The well-known solution of this problem is based on the use of contact forms (2) and the Cartan form, see e.g. [19], with

$$
\mathfrak{C}=\mathcal{L} \Omega+\rho_{\alpha}^{j} \omega^{\alpha} \wedge \Omega_{j}, \omega^{\alpha}=\mathrm{d} x^{\alpha}-x_{1_{i}}^{\alpha} \mathrm{d} X^{i}
$$

implying $\int_{S} j^{1}(\sigma)^{*} \mathfrak{L}=\int_{\mathcal{S}} j^{1}(\sigma)^{*} \mathfrak{C}$ as hor $(\mathfrak{C})=\mathfrak{L}$ is met, where the derivation of the functions $\rho_{\alpha}^{j}$ will be described next. Instead of (4) we consider

$$
\int_{\mathcal{S}} j^{1}(\sigma)^{*}\left(\mathrm{~L}_{j^{1}(\eta)}(\mathfrak{C})\right)=0
$$

and $\left.j^{1}(\eta)\right\rfloor \mathrm{d} \mathfrak{C}$ will deliver the domain conditions (pdes) and from $\left.j^{1}(\eta)\right\rfloor \mathfrak{C}$ the boundary expressions can be extracted, if we choose the functions $\rho_{\alpha}^{i}$ such that

$$
\left.\left.\operatorname{hor}\left(j^{1}(\eta)\right\rfloor \mathrm{d} \mathfrak{C}\right)=\operatorname{hor}(\eta\rfloor \mathrm{d} \mathfrak{C}\right)
$$

is met. Consequently,

$$
\left.\left.\operatorname{hor}\left(j^{1}(\eta)\right\rfloor \mathrm{d} \mathfrak{C}\right)=\operatorname{hor}\left(j^{1}(\eta)\right\rfloor\left(\mathrm{d} \mathcal{L} \wedge \Omega+\mathrm{d} \rho_{\alpha}^{j} \wedge \omega^{\alpha} \wedge \Omega_{j}-\rho_{\alpha}^{j} \mathrm{~d} x_{1_{j}}^{\alpha} \Omega\right)\right)
$$

follows, which can be evaluated as

$$
\left.\operatorname{hor}\left(j^{1}(\eta)\right\rfloor \mathrm{d} \mathfrak{C}\right)=\left(\partial_{\alpha} \mathcal{L} \eta^{\alpha}+\partial_{\alpha}^{1_{i}} \mathcal{L} \eta_{1_{i}}^{\alpha}-\rho_{\alpha}^{i} v_{1_{i}}^{\alpha}\right) \Omega-\operatorname{hor}\left(\mathrm{d} \rho_{\alpha}^{i}\right) \wedge \eta^{\alpha} \Omega_{i}
$$

where we have used the fact that hor $\left(\omega^{\alpha}\right)=0$ and $d_{1_{i}} \eta^{\alpha}=\eta_{1_{i}}^{\alpha}$. This expression (6) is independent of the jet-prolongation of the variational vector field $\eta_{1_{i}}^{\alpha}$ iff

$$
\rho_{\alpha}^{i}=\partial_{\alpha}^{1_{i}} \mathcal{L}
$$

such that

$$
\left.\operatorname{hor}\left(j^{1}(\eta)\right\rfloor \mathrm{d} \mathfrak{C}\right)=\eta^{\alpha}\left(\partial_{\alpha} \mathcal{L}-d_{1_{i}} \rho_{\alpha}^{i}\right) \Omega=\eta^{\alpha} \delta_{\alpha} \mathcal{L} \Omega
$$

holds, where we have used

$$
\operatorname{hor}\left(\mathrm{d} \rho_{\alpha}^{i}\right) \wedge \Omega_{i}=d_{1_{i}}\left(\rho_{\alpha}^{i}\right) \Omega=d_{1_{i}}\left(\partial_{\alpha}^{1_{i}} \mathcal{L}\right) \Omega .
$$

From (77) we see that we have recovered the Euler Lagrange operator that delivers the domain condition on $\mathcal{S}$, where $\delta_{\alpha}=\partial_{\alpha}-d_{1_{i}} \partial_{\alpha}^{1_{i}}$ denotes the variational derivative. 


\subsection{Boundary Conditions}

The boundary conditions on $\partial \mathcal{S}$ follow from

$$
\left.j^{1}(\eta)\right\rfloor \mathfrak{C}=\eta^{\alpha} \rho_{\alpha}^{i} \Omega_{i}=\eta^{\alpha}\left(\partial_{\alpha}^{1_{i}} \mathcal{L}\right) \Omega_{i} .
$$

Remark 2. It should be noted that the boundary conditions (8) on $\partial \mathcal{S}$ can be easily met, if one does not consider variations on the boundary, i.e. if $\eta$ vanishes. Conversely, arbitrary variations on $\partial \mathcal{S}$ lead to conditions for the first-order partial derivatives of $\mathcal{L}$. Furthermore, in the case of boundary control systems it also possible to extend the variational problem to include external boundary inputs that equate with $\partial_{\alpha}^{1_{i}} \mathcal{L}$ such that for arbitrary variations the boundary conditions are met.

The functions $\rho_{\alpha}^{j}$ in (5) have been derived in such a way that in the variational principle the domain integral becomes independent of derivatives of the variational field. Furthermore, $\rho_{\alpha}^{j}$ enter the boundary integral and determine the boundary conditions. As the choice for $\rho_{\alpha}^{j}$ is unique for first-order field theories, so are the expressions in the boundary integral.

To summarize, (4) for $k=1$ is equivalent to

$$
\int_{\mathcal{S}} j^{2}(\sigma)^{*}\left(\eta^{\alpha} \delta_{\alpha} \mathcal{L}\right) \Omega+\int_{\partial \mathcal{S}} j^{1}(\sigma)^{*}\left(\eta^{\alpha} \partial_{\alpha}^{1_{i}} \mathcal{L}\right) \Omega_{i}=0
$$

Remark 3. Alternatively, (9) can be derived using the integration by parts method in a straightforward and unique manner, as

$$
\begin{aligned}
\mathrm{L}_{j^{1}(\eta)}(\mathfrak{L}) & =\left(\eta^{\alpha} \partial_{\alpha} \mathcal{L}+\left(d_{1_{i}} \eta^{\alpha}\right) \partial_{\alpha}^{1_{i}} \mathcal{L}\right) \Omega \\
& =\eta^{\alpha}\left(\partial_{\alpha} \mathcal{L}-d_{1_{i}}\left(\partial_{\alpha}^{1_{i}} \mathcal{L}\right)\right) \Omega+d_{1_{i}}\left(\eta^{\alpha} \partial_{\alpha}^{1_{i}} \mathcal{L} \Omega\right)
\end{aligned}
$$

gives the same result as in (9) using Stokes' theorem. Thus, the Cartan form approach indeed qualifies for a coordinate free version of the integration by parts technique.

\subsection{Example}

Let us consider the simple model of a Timoshenko beam, see e.g. [14], where the dependent variables $x^{1}$ and $x^{2}$ correspond to the deflection of a cross section of the beam and the angle of rotation due to bending. We have that $m=2$ and $r=2$, such that $\mathcal{X}$ possesses the coordinates $\left(X^{1}, X^{2}, x^{1}, x^{2}\right)$ and the independent coordinates $\left(X^{1}, X^{2}\right)$ correspond to the time $X^{1}=t$ and a spatial coordinate $X^{2}=X$.

The Lagrangian density corresponding to the difference of the kinetic and potential energy densities is given as $\mathcal{L} \Omega$ with $\Omega=\mathrm{d} X^{1} \wedge \mathrm{d} X^{2}$ and

$$
\mathcal{L}=\frac{1}{2}\left(\rho\left(x_{10}^{1}\right)^{2}+J\left(x_{10}^{2}\right)^{2}-E I\left(x_{01}^{2}\right)^{2}-k A G\left(x_{01}^{1}-x^{2}\right)^{2}\right)
$$

where for simplicity we assume constant beam parameters $\rho, J, E, k, A, G$. Using (9) the pdes on the domain immediately follow as

$$
\begin{aligned}
& \rho x_{20}^{1}=k A G\left(x_{02}^{1}-x_{01}^{2}\right) \\
& J x_{20}^{2}=E I x_{02}^{2}+k A G\left(x_{01}^{1}-x^{2}\right),
\end{aligned}
$$

as the variational field $\eta$ is arbitrary on $\mathcal{S}$. The boundary expression follows from the boundary integral in (9) and leads to a condition of the form

$$
-\eta^{1} k A G\left(x_{01}^{1}-x^{2}\right)-\eta^{2} E I x_{01}^{2}
$$

which has to vanish at $X=0$ and $X=L$ (the spatial boundary) as in mechanics no variation on the time boundary takes place. For arbitrary $\eta^{1}$ and $\eta^{2}$ we derive the classical conditions that the shear force $k A G\left(x_{01}^{1}-x^{2}\right)$ and the bending moment $E I x_{01}^{2}$ have to vanish on the spatial boundary. Remark 4. As already pointed out in remark 2 it is possible to extend the variational problem, such that for external boundary inputs $Q(t)$ and $M(t)$ at $X=0$ and/or $X=L$ the boundary conditions read as $k A G\left(x_{01}^{1}-x^{2}\right)=Q$ and $E I x_{01}^{2}=M$. This can be achieved e.g. by considering an additive boundary integral in the variational principle such that external boundary inputs are incorporated. 


\section{Second-order Case}

Let us consider the case $\mathcal{L}=\mathcal{C}^{\infty}\left(\mathcal{J}^{2}(\mathcal{X})\right)$ and consequently the equation (4) for $k=2$. Again, using

$$
\left.\left.\mathrm{L}_{j^{2}(\eta)}(\mathfrak{L})=j^{2}(\eta)\right\rfloor \mathrm{d} \mathfrak{L}+\mathrm{d}\left(j^{2}(\eta)\right\rfloor \mathfrak{L}\right)
$$

we aim to decompose the variational problem into an expression on the domain and one on the boundary.

\subsection{The domain conditions}

Similar as in the first-order case we modify the Lagrangian density by extending it with contact forms of the type

$$
\omega_{I}^{\alpha}=\mathrm{d} x_{I}^{\alpha}-x_{I+1_{k}}^{\alpha} \mathrm{d} X^{k}, \quad 0 \leq \# I \leq 1 .
$$

Thus, we make the ansatz

$$
\mathfrak{C}=\mathcal{L} \Omega+\rho_{\alpha}^{j}\left(\mathrm{~d} x^{\alpha}-x_{1_{i}}^{\alpha} \mathrm{d} X^{i}\right) \wedge \Omega_{j}+\rho_{\alpha}^{1_{k}, j}\left(\mathrm{~d} x_{1_{k}}^{\alpha}-x_{1_{k}+1_{i}}^{\alpha} \mathrm{d} X^{i}\right) \wedge \Omega_{j}
$$

which reads as

$$
\mathfrak{C}=\mathcal{L} \Omega+\rho_{\alpha}^{j}\left(\mathrm{~d} x^{\alpha} \wedge \Omega_{j}-x_{1_{j}}^{\alpha} \Omega\right)+\rho_{\alpha}^{1_{k}, j}\left(\mathrm{~d} x_{1_{k}}^{\alpha} \wedge \Omega_{j}-x_{1_{k}+1_{j}}^{\alpha} \Omega\right) .
$$

To obtain the domain conditions in an analogous fashion as in the first-order case we compute $\left.\operatorname{hor}\left(j^{2}(\eta)\right\rfloor \mathrm{d} \mathfrak{C}\right)$ with

$$
\begin{aligned}
\mathrm{d} \mathfrak{C}= & \mathrm{d} \mathcal{L} \wedge \Omega+\mathrm{d} \rho_{\alpha}^{j} \wedge\left(\mathrm{d} x^{\alpha} \wedge \Omega_{j}-x_{1_{j}}^{\alpha} \Omega\right)-\rho_{\alpha}^{j} \mathrm{~d} x_{1_{j}}^{\alpha} \wedge \Omega \\
& +\mathrm{d} \rho_{\alpha}^{1_{k}, j} \wedge\left(\mathrm{d} x_{1_{k}}^{\alpha} \wedge \Omega_{j}-x_{1_{k}+1_{j}}^{\alpha} \Omega\right)-\rho_{\alpha}^{1_{k}, j} \mathrm{~d} x_{1_{k}+1_{j}}^{\alpha} \wedge \Omega
\end{aligned}
$$

and $\left.\operatorname{hor}\left(j^{2}(\eta)\right\rfloor \mathrm{d} \mathfrak{C}\right)$ can be stated as

$$
\left(\partial_{\alpha}^{I} \mathcal{L} \eta_{I}^{\alpha}-\rho_{\alpha}^{j} \eta_{1_{j}}^{\alpha}-\rho_{\alpha}^{1_{k}, j} \eta_{1_{k}+1_{j}}^{\alpha}\right) \Omega-\operatorname{hor}\left(\eta^{\alpha} \mathrm{d} \rho_{\alpha}^{j}+\eta_{1_{k}}^{\alpha} \mathrm{d} \rho_{\alpha}^{1_{k}, j}\right) \wedge \Omega_{j}
$$

with $0 \leq \# I \leq 2$ which simplifies to (6) in the first-order case. Hence, to cancel out the expressions that involve a jet-prolongation of the variational vector field $\eta_{I}^{\alpha}$, we have to choose for the case $\# I=2$

$$
\begin{aligned}
\rho_{\alpha}^{1_{k}, j}+\rho_{\alpha}^{1_{j}, k} & =\partial_{\alpha}^{1_{k}+1_{j}} \mathcal{L}, \quad k \neq j \\
\rho_{\alpha}^{1_{k}, k} & =\partial_{\alpha}^{1_{k}+1_{k}} \mathcal{L}
\end{aligned}
$$

and to guarantee that $\eta_{I}$ vanishes for the case $\# I=1$ we assign

$$
\rho_{\alpha}^{l}=\partial_{\alpha}^{1_{l}} \mathcal{L}-d_{1_{j}}\left(\rho_{\alpha}^{1_{l}, j}\right) .
$$

Finally,

$$
\left.\operatorname{hor}\left(j^{2}(\eta)\right\rfloor \mathrm{d} \mathfrak{C}\right)=\left(\partial_{\alpha} \mathcal{L}-d_{1_{j}}\left(\rho_{\alpha}^{j}\right)\right) \eta^{\alpha} \Omega
$$

where we have used the fact that all expressions $\eta_{I}$ for $\# I \neq 0$ are canceled out. Using (13) and (14) we obtain

$$
\partial_{\alpha} \mathcal{L}-d_{1_{l}}\left(\rho_{\alpha}^{l}\right)=\partial_{\alpha} \mathcal{L}-d_{1_{l}}\left(\partial_{\alpha}^{1_{l}} \mathcal{L}-d_{1_{j}}\left(\rho_{\alpha}^{1_{l}, j}\right)\right)
$$

and consequently

$$
\left.\operatorname{hor}\left(j^{2}(\eta)\right\rfloor \mathrm{d} \mathfrak{C}\right)=\delta_{\alpha} \mathcal{L} \eta^{\alpha} \Omega
$$


with

$$
\delta_{\alpha} \mathcal{L}=\sum_{\# I=0}^{2}(-1)^{\# I} d_{I} \partial_{\alpha}^{I} \mathcal{L} .
$$

Hence, the domain conditions involve again the variational derivatives (the components of the EulerLagrange operator) (15) which is of second-order in this case. The preceding calculation verifies that despite of the fact that the choice of contact forms in (13) is not unique, it has no influence on the pdes, which is a well-known fact. But this non uniqueness will have an impact on the boundary conditions as demonstrated in the following.

\subsection{The boundary conditions}

From (13) it is obvious that there is no unique choice for the functions $\rho_{\alpha}^{1_{k}, j}$ when $k \neq j$ which has the consequence that in the boundary expression, reading as

$$
\left.\left.j^{2}(\eta)\right\rfloor \mathfrak{C}=j^{1}(\eta)\right\rfloor \mathfrak{C}=\left(\rho_{\alpha}^{i} \eta^{\alpha}+\rho_{\alpha}^{1_{k}, i} \eta_{1_{k}}^{\alpha}\right) \Omega_{i},
$$

a different choice for $\rho_{\alpha}^{1_{k}, i}$ leads to different boundary conditions. To overcome this problem we pose an extra condition on the set of possible boundary terms in order to extract the physical meaningful boundary terms. To this end, we assume that the boundary of the submanifold $\mathcal{S}$ which is denoted as $\partial \mathcal{S}$ is parameterized as $X^{r}=$ const. such that the coordinates for $\mathcal{D}$ are $\left(X_{\partial}^{i}, X^{r}\right)$ with $X_{\partial}^{i}=\left(X^{1}, \ldots, X^{r-1}\right)$ which is possible at least locally by a change of the independent coordinates, such that in these coordinates adapted to the boundary the boundary volume form $\left.\Omega_{\partial}=\partial_{r}\right\rfloor \Omega$ can be introduced. It is now remarkable that for a section $\sigma_{\partial}$ on the boundary, i.e. $x^{\alpha}=\sigma_{\partial}^{\alpha}\left(X_{\partial}^{i}\right)$, we can compute all the first-order jet-variables apart from the $X^{r}$ direction, which represents an additional degree of freedom on the boundary.

Remark 5. Let us consider the inclusion mapping $\iota: \partial \mathcal{D} \rightarrow \mathcal{D}$ in adapted coordinates. Then $\iota^{*}(\mathcal{X})$ and $\iota^{*}\left(\mathcal{J}^{1}(\mathcal{X})\right)$ are equipped with induced coordinates $\left(X_{\partial}^{i}, x^{\alpha}\right)$ and $\left(X_{\partial}^{i}, x^{\alpha}, x_{1_{l}}^{\alpha}\right), l=1, \ldots, r$, respectively. It is worth noting that $\iota^{*}\left(\mathcal{J}^{1}(\mathcal{X})\right)$ does not qualify as a jet-manifold as we have too few independent coordinates, but the coordinate $x_{1_{r}}^{\alpha}$ will play an essential role in deriving the boundary conditions. This construction has been used also in [3] to introduce the so-called extended Cartan form on the boundary. We will pursue a different target, namely we will exploit this boundary structure to give conditions for the choice of the under determined functions $\rho_{\alpha}^{1_{k}, i}$.

The boundary conditions are derived by means of

$$
\left.j^{1}(\eta)\right\rfloor \mathfrak{C}=\left(\rho_{\alpha}^{r} \eta^{\alpha}+\rho_{\alpha}^{1_{k}, r} \eta_{1_{k}}^{\alpha}\right) \Omega_{\partial}=\left(\rho_{\alpha}^{r} \eta^{\alpha}+\rho_{\alpha}^{1_{1}, r} \eta_{1_{1}}^{\alpha}+\ldots+\rho_{\alpha}^{1_{r}, r} \eta_{1_{r}}^{\alpha}\right) \Omega_{\partial}
$$

where the summation is carried out over $k$ as $r$ is fixed in the adapted coordinates. On the boundary, independent variations with respect to $x^{\alpha}$ and $x_{1_{r}}^{\alpha}$ can be carried out, as discussed above, such that we are led to set

$$
\rho_{\alpha}^{1_{k}, r}=0, \quad k=1, \ldots, r-1
$$

such that

$$
\left.j^{1}(\eta)\right\rfloor \mathfrak{C}=\left(\rho_{\alpha}^{r} \eta^{\alpha}+\rho_{\alpha}^{1_{r}, r} \eta_{1_{r}}^{\alpha}\right) \Omega_{\partial}
$$

is met.

Remark 6. From a variational point of view $\rho_{\alpha}^{1_{k}, r}=0, k \neq r$ guarantees that only independent variations arise in the boundary integral, which can be interpreted as an additional postulate which selects the appropriate Cartan form out of a set of possible Cartan forms that all deliver the same domain conditions. It should be noted that this choice always leads to the minimal possible boundary conditions. The coordinate representation is well justified in adapted coordinates only, i.e. when the boundary is parameterized as $X^{r}=$ const.

This means that in the relations (13) we have to choose $\rho_{\alpha}^{1_{r}, k}=\partial_{\alpha}^{1_{r}+1_{k}} \mathcal{L}$. From relation (14) we observe that then the coefficient of $\eta^{\alpha}$ namely $\rho_{\alpha}^{r}$ which is

$$
\rho_{\alpha}^{r}=\partial_{\alpha}^{1_{r}} \mathcal{L}-d_{1_{k}}\left(\rho_{\alpha}^{1_{r}, k}\right) .
$$


involves exactly the terms $\rho_{\alpha}^{1_{r}, k}$. Hence we have exploited the degree of freedom regarding the functions $\rho_{\alpha}^{1_{k}, i}$ in (13) to generate the admissible boundary expressions.

Remark 7. It should be noted that in [3] a different strategy is suggested which requires an additional integration by parts or in terms of [3] an extended Cartan form which contains an additive total differential which corrects the wrong boundary terms. E.g. an expression involving $\eta_{1_{k}}^{\alpha}$ for $1_{k} \neq 1_{r}$ can be again integrated by parts to produce an admissible $\eta^{\alpha}$ term. Both strategies give the same boundary conditions as the additional integration by parts exactly delivers the second term in the right hand side of (18).

\section{Examples for second-order mechanical systems}

In this section we demonstrate our approach by means of two examples - the Euler-Bernoulli beam and the Kirchhoff plate.

\subsection{Euler-Bernoulli Beam}

We have two independent variables $X^{1}=t$ and $X^{2}=X$, hence $\operatorname{dim}(\mathcal{D})=2$, where $t$ is the time coordinate and $X$ the spatial variable (we set $\mathcal{S}=\mathcal{D}$ for simplicity). The dependent variable is the deflection $x^{1}$, such that $\mathcal{X}$ is equipped with the coordinates $\left(X^{1}, X^{2}, x^{1}\right)$ and $\Omega=\mathrm{d} X^{1} \wedge \mathrm{d} X^{2}=$ $\mathrm{d} t \wedge \mathrm{d} X$ together with the Lagrangian (kinetic minus potential energy)

$$
\mathcal{L}=\frac{1}{2} \rho A\left(x_{10}^{1}\right)^{2}-\frac{1}{2} E I\left(x_{02}^{1}\right)^{2}
$$

where again for simplicity we consider constant physical parameters $\rho, A, E, I$. The pdes follow from the Euler-Lagrange operator (15), which in this case simplifies to $\delta_{1}=-d_{10} \partial_{1}^{10}+d_{02} \partial_{1}^{02}$ such that we obtain the well-known pde

$$
\rho A x_{20}^{1}+E I x_{04}^{1}=0 .
$$

\subsubsection{Boundary Conditions}

To obtain the boundary conditions, we consider the relation (16) with $\left.\Omega_{\partial}=\Omega_{r}=\partial_{2}\right\rfloor \Omega=-\mathrm{d} t$ since we are interested in the spatial boundary (no variation on the time boundary) and derive

$$
\left(\rho_{1}^{2} \eta^{1}+\rho_{1}^{10,2} \eta_{10}^{1}+\rho_{1}^{01,2} \eta_{01}^{1}\right) \Omega_{\partial}=0
$$

As no mixed partial derivatives appear in $\mathcal{L}, \rho_{1}^{10,2}=0$ is met automatically and we have

$$
\begin{aligned}
\rho_{1}^{01,2} & =\partial_{1}^{02} \mathcal{L}=-E I x_{02}^{1} \\
\rho_{1}^{2} & =-d_{01} \rho_{1}^{01,2}=E I x_{03}^{1}
\end{aligned}
$$

such that we obtain the conditions on the spatial boundary as

$$
\eta^{1} E I x_{03}^{1}=0, \quad \eta_{01}^{1} E I x_{02}^{1}=0 .
$$

Here, $E I x_{03}^{1}$ corresponds to the shear force and $E I x_{02}^{1}$ to the bending moment and $\eta^{1}$ and $\eta_{01}^{1}$ take their values according to the support on the boundary.

Remark 8. Let us consider a cantilever beam that is built-in at $X=0$ (clamped end) and free at $X=L$ (free end). Then at $X=0$ the imposed geometric boundary conditions imply $\eta^{1}=\eta_{01}^{1}=0$ whereas at $X=L$ we have the dynamic boundary conditions $E I x_{02}^{1}=E I x_{03}^{1}=0$. Furthermore, it is again possible to apply external forces and torques at $X=L$ which can be included in the variational principle.

As no mixed partial derivatives appear in this example, the Cartan form approach is unambiguous although the example belongs to second-order theory. This will be different for the following example. 


\subsection{Kirchhoff Plate}

Let us consider the case of a (rectangular) Kirchhoff plate with three independent variables $X^{1}=$ $t, X^{2}=X$ and $X^{3}=Y$, such that we have two spatial variables and the time. Hence, $\mathcal{X}$ is equipped with the coordinates $\left(X^{1}, X^{2}, X^{3}, x^{1}\right)$ where $x^{1}$ denotes the deflection. The Lagrangian is given as $\mathcal{L}=\mathcal{T}-\mathcal{V}$ with $2 \mathcal{T}=\left(x_{100}^{1}\right)^{2}$ and

$$
2 \mathcal{V}=\left(x_{020}^{1}\right)^{2}+\left(x_{002}^{1}\right)^{2}+2 \nu x_{020}^{1} x_{002}^{1}+2(1-\nu)\left(x_{011}^{1}\right)^{2}
$$

where we have set the physical parameters to one apart from the Poisson's ratio $\nu$. Again the pdes follow from the Euler-Lagrange operator (15), with

$$
\delta_{1}=-d_{100} \partial_{1}^{100}+d_{020} \partial_{1}^{020}+d_{002} \partial_{1}^{002}+d_{011} \partial_{1}^{011}
$$

such that we obtain

$$
x_{200}^{1}+x_{040}^{1}+x_{004}^{1}+2 x_{022}^{1}=0 .
$$

\subsubsection{The boundary conditions}

To obtain the boundary conditions on the boundary $X^{3}=Y=$ const. we consider

$$
\left(\rho_{1}^{3} \eta^{1}+\rho_{1}^{010,3} \eta_{010}^{1}+\rho_{1}^{001,3} \eta_{001}^{\alpha}\right) \Omega_{\partial}
$$

where we already have used $\rho_{1}^{100,3}=0$ since no mixed time and spatial derivatives appear. The non-zero components for $\rho_{1}$ of regarding the jet-order $\# I=2$ in $\mathcal{L}$ are

$$
\rho_{1}^{001,3}=\partial_{1}^{002} \mathcal{L}, \quad \rho_{1}^{010,2}=\partial_{1}^{020} \mathcal{L}
$$

as well as

$$
\rho_{1}^{010,3}+\rho_{1}^{001,2}=\partial_{1}^{011} \mathcal{L}
$$

It is remarkable that for the mixed spatial derivative we get two possible contact forms. For \#I=1 we obtain from $\rho_{\alpha}^{l}=\partial_{\alpha}^{1_{l}} \mathcal{L}-d_{1_{j}}\left(\rho_{\alpha}^{1_{l}, j}\right)$ the coordinate expressions

$$
\begin{aligned}
& \rho_{1}^{3}=\partial_{1}^{001} \mathcal{L}-d_{001} \rho_{1}^{001,3}-d_{010} \rho_{1}^{001,2} \\
& \rho_{1}^{2}=\partial_{1}^{010} \mathcal{L}-d_{010} \rho_{1}^{010,2}-d_{001} \rho_{1}^{010,3}
\end{aligned}
$$

and $\rho_{1}^{1}=\partial_{1}^{100} \mathcal{L}$. Since $\eta_{010}^{1}$ is not admissible on the boundary of interest we can choose $\rho_{1}^{010,3}=0$ and consequently $\rho_{1}^{001,2}=\partial_{1}^{011} \mathcal{L}$ follows. Hence,

$$
\rho_{1}^{3}=-d_{001} \rho_{1}^{001,3}-d_{010} \rho_{1}^{001,2}=\left(x_{003}^{1}+(2-\nu) x_{021}^{1}\right)
$$

and therefore on the spatial boundary $X^{3}=Y=$ const. we obtain (no variation on the time boundary)

$$
\begin{aligned}
\eta^{1}\left(x_{003}^{1}+(2-\nu) x_{021}^{1}\right) & =0 \\
-\eta_{001}^{1}\left(x_{002}^{1}+\nu x_{020}^{1}\right) & =0
\end{aligned}
$$

where (22) is a condition for the shear force and (23) for the bending moment. As a consequence, we obtain two boundary conditions, whereas a wrong choice for $\rho_{1}$ (corresponding to a wrong integration by parts) would deliver three (compare with (20)). To construct the boundary conditions for the boundary $X^{2}=X=$ const. one can easily adopt the procedure or re-parameterize the independent coordinates. Furthermore, it should be noted that in an analogous fashion as in remark 8 the introduction of imposed geometric and dynamic boundary conditions can be accomplished. 


\section{Boundary Ports}

To motivate for the idea of boundary ports, let et us consider a Hamiltonian of first-order based on an underlying bundle structure $\mathcal{X} \rightarrow \mathcal{D}$. Hence, we have $\mathfrak{H}=\mathcal{H} \Omega$ with $\mathcal{H} \in C^{\infty}\left(\mathcal{J}^{1}(\mathcal{X})\right)$. Depending on a chosen section $s: \mathcal{D} \rightarrow \mathcal{X}$ we are led to compute $H(s)=\int_{\mathcal{D}} j^{1}(s)^{*}(\mathfrak{H})$. Let us consider now an evolutionary vector-field $v=v^{\alpha} \partial_{\alpha}$, corresponding to the pdes

$$
\dot{x}^{\alpha}=v^{\alpha}, \quad v^{\alpha} \in C^{\infty}\left(\mathcal{J}^{2}(\mathcal{X})\right)
$$

and we are interested in the change of $H$ along the section $s$, where $s$ is a formal solution of the pdes parameterized in $t$, i.e.

$$
\partial_{t} s(X)=v\left(X, \partial_{J} s(X)\right), \quad \# J \leq 2
$$

together with appropriate boundary conditions, where we have suppressed the explicit dependence of $s$ with respect to the evolution parameter and well-posedness of the problem is assumed.

The idea of ports in a Hamiltonian setting can be visualized by computing a power balance relation by means of the Hamiltonian density which can be interpreted as an energy density in many applications. Thus we evaluate

$$
\dot{H}=\partial_{t} H(s)=\int_{\mathcal{S}} j^{2}(s)^{*}\left(\mathrm{~L}_{j^{1}(v)} \mathfrak{H}\right)
$$

and regarding the evaluation of the expression $\mathrm{L}_{j^{1}(v)} \mathfrak{H}$ appearing in (25) the same reasoning as in section 3 can be applied and

$$
\partial_{t} H(s)=\int_{\mathcal{S}} j^{2}(s)^{*}\left(v^{\alpha}\left(\delta_{\alpha} \mathcal{H}\right) \Omega\right)+\int_{\partial \mathcal{S}} j^{1}(s)^{*}\left(v^{\alpha}\left(\partial_{\alpha}^{1_{i}} \mathcal{H}\right) \Omega_{i}\right)
$$

follows which is the counterpart to (9).

Remark 9. It should be noted that instead of a variational vector field $\eta$ that performs the variation, now an evolutionary vector field that corresponds to a dynamical systems is applied. But form a mathematical point of view the calculations remain valid when $\mathcal{L}$ is replaced by $\mathcal{H}$ and $\eta$ is replaced by $v$.

Thus, we have obtained a power balance relation when $\mathfrak{H}=\mathcal{H} \Omega$ corresponds to an energy density. Obviously, in this power balance relation we have a contribution in the domain and one on/over the boundary. We can restate (26) to obtain

$$
\left.\left.\dot{H}=\int_{\mathcal{S}} j^{2}(s)^{*}(v\rfloor \delta \mathfrak{H}\right)+\int_{\partial \mathcal{S}} j^{1}(s)^{*}(v\rfloor \delta^{\partial} \mathfrak{H}\right)
$$

with $\delta \mathfrak{H}=\left(\delta_{\alpha} \mathcal{H}\right) \mathrm{d} x^{\alpha} \wedge \Omega$ and $\delta^{\partial} \mathfrak{H}=\left(\partial_{\alpha}^{1_{i}} \mathcal{H}\right) \mathrm{d} x^{\alpha} \wedge \Omega_{i}$. Hence, choosing

$$
v=(\mathcal{J}-\mathcal{R})(\delta \mathfrak{H})+u\rfloor \mathcal{G}
$$

where $\mathcal{J}$ is a skew- symmetric map and $\mathcal{R}$ is positive semi-definite, we can express the impact on the domain as

$$
\left.\left.\int_{\mathcal{S}} j^{2}(s)^{*}(-\mathcal{R}(\delta \mathfrak{H})\rfloor \delta \mathfrak{H}+y\right\rfloor u\right)=\int_{\mathcal{S}} j^{2}(s)^{*}\left(-\left(\delta_{\alpha} \mathcal{H}\right) \mathcal{R}^{\alpha \beta}\left(\delta_{\beta} \mathcal{H}\right)+y_{\xi} u^{\xi}\right) \Omega
$$

with $y=\mathcal{G}^{*}(\delta \mathfrak{H})$ and the input map $\mathcal{G}$ together with its adjoint $\mathcal{G}^{*}$. The boundary impact (depending on the boundary conditions of the pdes) is determined by the expression $v\rfloor \delta^{\partial} \mathfrak{H}$.

Corollary 10. Given evolutionary pdes of the form (24) with the generalized vector field $v$ as in (28), the formal change of the first-order Hamiltonian $H$ along solutions of 24) can be decomposed in a dissipative term in the domain $-\mathcal{R}(\delta \mathfrak{H})\rfloor \delta \mathfrak{H}$, in a power port in the domain $y\rfloor u$ and in a power port on the boundary $v\rfloor \delta^{\partial} \mathfrak{H}$. 
The representation (28) together with the collocated output $y=\mathcal{G}^{*}(\delta \mathfrak{H})$ can be seen as a generalization of the classical port-Hamiltonian formulation of ODEs, see e.g. [26], to the infinitedimensional case based on the underlying jet-bundle structure and has been proposed and discussed in [20, 24, 25]. The power balance relation (29) is valid when the maps $\mathcal{J}, \mathcal{R}$ and $\mathcal{G}$ do not involve differential operators. However, also this differential operator case can be discussed in the proposed setting, see e.g. [25], but then additional boundary expressions have to be taken into account due to the differential operators. The first-order case can be also handled by using the classical integration by parts technique, as it has been done in the cited references. For second-order theories similar problems as in the Lagrangian setting (calculus of variations) appear and we will show that the proposed Cartan form approach can also be exploited in the port-Hamiltonian setting for the determination of the boundary ports in a similar way as it has been used in the Lagrangian scenario to determine the boundary conditions.

\subsection{Domain and Boundary Impact for the second-order case}

Let us consider a Hamiltonian density $\mathfrak{H}=\mathcal{H} \Omega$ with $\mathcal{H} \in C^{\infty}\left(\mathcal{J}^{2}(\mathcal{X})\right)$ and an evolutionary vector field $v$. Then, to evaluate

$$
\dot{H}=\partial_{t} H(s)=\int_{\mathcal{S}} j^{4}(s)^{*}\left(\mathrm{~L}_{j^{2}(v)} \mathfrak{H}\right)
$$

we follow the same consideration as in section 4 by augmenting $\mathcal{H} \Omega$ with appropriate contact forms. This immediately gives us the desired decomposition of $\dot{H}$ into a domain expression $\dot{H}_{\mathcal{S}}$ and a boundary expression $\dot{H}_{\partial \mathcal{S}}$ with

$$
\left.\dot{H}_{\mathcal{S}}=\int_{\mathcal{S}} j^{4}(s)^{*}(v\rfloor \delta \mathfrak{H}\right)
$$

where $\delta$ involves the Euler-Lagrange operator of second-order, i.e.

$$
\delta_{\alpha} \mathcal{H}=\sum_{\# I=0}^{2}(-1)^{\# I} d_{I} \partial_{\alpha}^{I} \mathcal{H} .
$$

Thus, again the consideration of (28), where $\delta \mathfrak{H}$ involves (30) in this case, gives an analogous power balance relation. The crucial point is again the boundary impact $\dot{H}_{\partial \mathcal{S}}$ and the key to solve the problem is to consider adapted coordinates with respect to the boundary such that

$$
\dot{H}_{\partial \mathcal{S}}=\int_{\partial \mathcal{S}} j^{3}(s)^{*}\left(\rho_{\alpha}^{r} v^{\alpha}+\rho_{\alpha}^{1_{r}, r} v_{1_{r}}^{\alpha}\right) \Omega_{\partial}
$$

can be stated (which is the counterpart to (17)), where the determination of the function $\rho_{\alpha}^{r}$ and $\rho_{\alpha}^{1_{r}, r}$ follows from (13) and (14) by substituting $\mathcal{L}$ with $\mathcal{H}$.

\subsection{Example}

The port-Hamiltonian representation of the Kirchhoff plate can be accomplished by choosing a manifold $\mathcal{X}$ equipped with the coordinates $\left(X^{1}, X^{2}, x^{1}, p_{1}\right)$ where $x^{1}$ again denotes the deflection and $x^{2}=p_{1}$ the linear momentum It should be noted that the number of independent variables is two, in contrast to section 5.2 as the time is serving as an evolution parameter. The Hamiltonian is given as $\mathcal{H}=\mathcal{T}+\mathcal{V}$ with $2 \mathcal{T}=\left(p_{1}\right)^{2}$ and

$$
2 \mathcal{V}=\left(x_{20}^{1}\right)^{2}+\left(x_{02}^{1}\right)^{2}+2 \nu x_{20}^{1} x_{02}^{1}+2(1-\nu)\left(x_{11}^{1}\right)^{2} .
$$

The pdes read as

$$
\left[\begin{array}{l}
\dot{x}^{1} \\
\dot{p}_{1}
\end{array}\right]=\left[\begin{array}{cc}
0 & 1 \\
-1 & 0
\end{array}\right]\left[\begin{array}{c}
\delta_{1} \mathcal{H} \\
\delta^{1} \mathcal{H}
\end{array}\right], \quad \mathcal{J}=\left[\begin{array}{cc}
0 & 1 \\
-1 & 0
\end{array}\right]
$$

with

$$
\delta^{1}=\frac{\partial}{\partial p_{1}}, \quad \delta_{1}=\partial_{1}-d_{1_{i}} \partial_{1}^{1_{i}}+d_{J} \partial_{1}^{J}, \quad \# J=2
$$


which is

$$
\dot{x}^{1}=p_{1}, \quad \dot{p}_{1}=-\left(x_{40}^{1}+x_{04}^{1}+2 x_{22}^{1}\right) .
$$

The Hamiltonian includes second-order jet variables, and the application of the variational derivative implies then forth-order pdes in our evolutionary setting.

Remark 11. The pdes correspond to (19) where the second time derivative, i.e. $x_{200}$, is written as a first-order derivative with respect to the momentum variable $p_{1}$ to obtain an evolutionary representation in the Hamiltonian setting. Furthermore, it is easily checked that (32) is of the form $v=(\mathcal{J}-\mathcal{R})(\delta \mathfrak{H})+u\rfloor \mathcal{G}$ with $\mathcal{R}=0$ and $\mathcal{G}=0$ and with the second-order variational derivatives $\delta^{1}$ and $\delta_{1}$ acting on $\mathcal{H}$, where $\delta^{1}$ degenerates to a partial derivative as no jet-variables with respect to $p_{1}$ are involved.

Next we wish to analyze the power balance relation in order to obtain the corresponding ports. As we use a skew-symmetric map $\mathcal{J}$ we can conclude that $\dot{H}_{\mathcal{D}}$ in $\dot{H}=\dot{H}_{\mathcal{D}}+\dot{H}_{\partial \mathcal{D}}$ is equal to zero and that only a boundary impact has to be considered.

\subsubsection{The boundary impact}

From (31) we obtain on the boundary $X^{2}=$ const. the expression

$$
\dot{H}_{\partial \mathcal{D}}=-\int\left(v^{1} \rho_{1}^{2}+v_{01}^{1} \rho_{1}^{01,2}\right) \mathrm{d} X^{1}=\int\left(\dot{x}^{1} F_{S}+\dot{x}_{01}^{1} M_{B}\right) \mathrm{d} X^{1}
$$

with the shear force $F_{S}=\left(x_{03}^{1}+(2-\nu) x_{21}^{1}\right)$ appearing in the power port $\dot{x}^{1} F_{S}$ and the bending moment $M_{B}=-\left(x_{02}^{1}+\nu x_{20}^{1}\right)$ in $\dot{x}_{01}^{1} M_{B}$. The collocated quantities are $\dot{x}^{1}=v^{1}$ (velocity) and $\dot{x}_{01}^{1}=v_{01}^{1}$ (angular velocity). Hence, to obtain the physically correct ports, it is vitally important to perform the integration by parts properly, which in our language amounts for a correct selection of the coefficients of the Cartan-form in accordance with the parameterization of the boundary.

\section{Conclusion}

In this contribution we have provided a strategy to compute the boundary conditions for secondorder field theories in a variational formulation in an algorithmic fashion suitable for the computer algebra implementation. Furthermore, for the class of port-Hamiltonian systems formulated on jetbundles, the same reasoning regarding a minimal number of boundary ports can be carried out, as the mathematical problem of computing the variation of the Lagrangian density is equivalent to the evaluation of the change of the Hamiltonian (the total energy) along solutions of an evolutionary set of pdes in a port-Hamiltonian setting.

\section{References}

[1] V. Aldaya and J. A. de Azcarraga. Variational principles on $r$-th order jets of fiber bundles in field theory. J. Mathematical Phys., 19:1869-1875, 1978.

[2] C. Campos, M. de Leon, M. de Diego, and J. Vankerschaver. Unambiguous formalism for higher order lagrangian field theories. Journal of Physics A: Mathematical and Theoretical, 42(47):475207, 2009.

[3] H. Ennsbrunner. Infinite-dimensional Euler-Lagrange and Port Hamiltonian Systems. University Linz, PhD-Thesis, 2006.

[4] H. Ennsbrunner and K. Schlacher. On the geometrical representation and interconnection of infinite dimensional port controlled hamiltonian systems. In Proceedings IEEE Conference on Decision and Control and European Control Conference (CDC-ECC), pages 5263-5268, 2005.

[5] P. L. Garcia Pérez and J. Muñoz Masqué. Higher order regular variational problems, pages 136-159. Symplectic Geometry and Mathematical Physics. Birkhäuser, Boston, 1991. 
[6] G. Giachetta, L. Mangiarotti, and G. Sardanashvily. New Lagrangian and Hamiltonian Methods in Field Theory. World Scientific, Singapore, 1997.

[7] M. J. Gotay. An exterior differential systems approach to the Cartan form, pages 160-188. Symplectic Geometry and Mathematical Physics. Birkhäuser, Boston, 1991.

[8] M.J. Gotay. A multisymplectic framework for classical field theory and the calculus of variations ii: Space + time decomposition. Differential Geometry and its Applications, (1):375-390, 1991.

[9] S. Kouranbaeva and S. Shkoller. A variational approach to second-order multisymplectic field theory. Journal of Geometry and Physics, 35:333-366, 2000.

[10] D. Krupka. On the local structure of the Euler-Lagrange mapping of the calculus of variations. Proceedings of the Conference on Differential Geometry and its Applications, Sept. 1980, Universita Karlova, 1981.

[11] A. Macchelli, A.J. van der Schaft, and C. Melchiorri. Port hamiltonian formulation of infinite dimensional systems i. modeling. In Proceedings 43rd IEEE Conference on Decision and Control $(C D C)$, pages $3762-3767,2004$.

[12] A. Macchelli, A.J. van der Schaft, and C. Melchiorri. Port hamiltonian formulation of infinite dimensional systems ii. boundary control by interconnection. In Proceedings 43rd IEEE Conference on Decision and Control (CDC), pages 3768-3773, 2004.

[13] B. Maschke and A.J. van der Schaft. Compositional Modelling of Distributed-Parameter Systems, pages 115-154. Advanced Topics in Control Systems Theory. Springer Lecture Notes in Control and Information Sciences, London, 2005.

[14] L. Meirovitch. Principles and Techniques of Vibrations. Prentice Hall, New Jersey, 1997.

[15] G. Moreno and M.E. Stypa. Natural boundary conditions in geometric calculus of variations. Mathematica Slovaca, 65(6):1531-1556, 2015.

[16] P.J. Olver. Applications of Lie Groups to Differential Equations. Springer-Verlag, New York, 1986.

[17] P. D. Prieto-Martínez and N. Román-Roy. A new multisymplectic unified formalism for secondorder classical field theories. Journal of Geometric Mechanics, 7:203-253, 2015.

[18] D. J. Saunders and M. Crampin. On the legendre map in higher-order field theories. J. Phys. A: Math. Gen., 23:3169-3182, 1990.

[19] D.J. Saunders. The Geometry of Jet Bundles. Cambridge University Press, Cambridge, 1989.

[20] K. Schlacher. Mathematical modeling for nonlinear control: a hamiltonian approach. Mathematics and Computers in simulation, 97:829-849, 2008.

[21] M. Schöberl and K. Schlacher. First order hamiltonian field theory and mechanics. Mathematical and Computer Modelling of Dynamical Systems, 17:105-121, 2011.

[22] M. Schöberl and K. Schlacher. Lagrangian and port-hamiltonian formulation for distributedparameter systems. In Proceedings, 8th Mathmod Conference, Vienna, Austria, pages 610-615, 2015.

[23] M. Schöberl and A. Siuka. On the port-hamiltonian representation of systems described by partial differential equations. In Proceedings 4th IFAC Workshop on Lagrangian and Hamiltonian Methods for Non Linear Control, pages 1-6, 2012.

[24] M. Schöberl and A. Siuka. On casimir functionals for infinite-dimensional port-hamiltonian control systems. IEEE Transactions on Automatic Control, 58:1823-1828, 2013. 
[25] M. Schöberl and A. Siuka. Jet bundle formulation of infinite-dimensional port-hamiltonian systems using differential operators. Automatica, 50:607-613, 2014.

[26] A.J. van der Schaft. L2-Gain and Passivity Techniques in Nonlinear Control. Springer-Verlag, New York, 2000.

[27] A.J. van der Schaft and B.M. Maschke. Hamiltonian formulation of distributed-parameter systems with boundary energy flow. Journal of Geometry and Physics, 42:166-194, 2002. 